\section{Shroud dating still questioned}

SIR-Damon et al. (Nature 337, 611-615; 1989) asserted that radiocarbon dating performed in 1988 provided conclusive evidence that the linen of the Shroud of Turin was mediaeval. However, most of the scientists involved in the studies on the Shroud clearly showed at the Paris International Symposium (7-8 September 1989) that they utterly disagreed with the conclusions of this article; the main reason was the lack of reliability of the results due to several methodological inadequacies. On a matter of such wide interest, it is important that the scientific community should be seen to come to a definitive conclusion about the value of the published dates. For this reason I would like to re-open the debate.

As a matter of fact, survey protocols have to be performed according to a method capable of avoiding investigator bias, in order to achieve relevant and accepted conclusions (F. Ederer Amer. $J$. Med. 58, 295-299; 1975). In the case of the radiocarbon dating of the Shroud, the procedures were neither blind nor controlled, contrary to the assertions of M. S. Tite (Nature 332, 482; 1988). As a result, the following questions have to be asked: (1) What were the scientific reasons for abandoning the blind procedure and the full documentation by video film and photography (I showed at the Cagliari conference that a true blind radiocarbon dating was feasible)? (2) What was the methodological need for giving the ages of the control tissues to the laboratories before

\section{Complexity levels}

SiR-P. R. Sheldon's question on complexity (Nature 347, 704; 1990) can be addressed at several levels. More complex organisms can generate more diverse "progeny' because they have more discrete parts that can be modified to form distinct variants of the parental type. Complexity breeds complexity as a power function of the number of discrete subunits. Plants, for example, which have many fewer distinct cell types than animals, show considerably less diversity. Arthropods modify their many pairs of limbs for more distinct functions than vertebrates, which have but two pairs. Complexity is a 'runaway' phenomenon only in the sense of its exponential potential for creating diversity.

Simple and complex organisms coexist stably through time because of ecological tradeoffs in capturing energy. Complexity requires larger body size, which reduces the rate of reproduction and the number of individuals that can be sustained on a unit of resource - but larger size enables individuals to live longer because of increased physiological buffering. the radiocarbon dating procedure? (3) What were the detailed data of the carbon measurements in each series of analysis? (4) What were the detailed data of the statistical analysis supervised by the " $G$. Colonetti" Institute? (5) What was the scientific reason for asserting without any discussion that the results obtained provided conclusive evidence that the linen of the Shroud was mediaeval, whereas it is in complete disagreement with every result obtained previously by scientists in the past 90 years?

All these important questions should have been discussed at the scientific conference held in Cagliari (29-30 April 1990), since its topic was precisely the Shroud dating. It was reasonable to expect some of the scientists involved in the dating performed in 1988 to attend. And above all, the paper to be presented by Professor Hall on "An attempt to answer criticisms concerning the dating of the Shroud" was eagerly anticipated. Unfortunately, none of the 21 authors of the article quoted previously was present in Cagliari, including Hall.

So, the international community of scientists interested in research on the Turin Shroud is still awaiting answers from Hall and Tite to these questions.

OLIVIER POURRAT

Clinique Médicale,

Université de Poitiers,

Hôpital Jean-Bernard,

BP 577 ,

86021 Poiters, France

Although larger organisms cannot outreproduce smaller ones, they can compete with them simply by eating them. Being fast-reproducing and ubiquitous as well as simple and small is a good strategy too.

Eukaryotic cells are more complex than prokaryotic ones because they can become enormously larger in size and truly multicellular (in part because of a nuclear membrane). The eukaryotic lineage has evolved motility within single cells, enabling cells to move their membranes about, internalizing some bits and returning others to the surface during ingestion of food and ejection of wastes. By these processes, eukaryotes overcame the restriction that volume rapidly outstrips surface area, a physical law that keeps prokaryotes small.

The active cytoplasm presumably selected for a nuclear membrane to protect its active DNA from being broken apart by its streaming cytoplasm. The nuclear membrane coincidentally separated the processes of transcription and translation. Only messenger RNA that moved out of the nucleus and into the cytoplasm could then be translated into structural or enzymatic proteins. If the

\section{Man's inhumanity}

SIR-Brown and Maroudas (Nature $\mathbf{3 4 8}$, $669 ; 1990$ ) bemoan the passing of the good old days when British science was run on the "well tried and trusted principles" of "backing the man". Well, yes, I deeply sympathize. But how simple, how effortless, it would have been to have said "backing the individual". Of course, everyone knows that man means woman as well, and that only a woman could be so small-minded as to think that it mattered. But perhaps their insensitive phraseology is evidence of a deeper unwillingness to recognize and make concessions to social change? For example, to look around and wonder why it is that there are so few women in positions of influence in British science compared with the United States. Rather than wringing their hands over a hard-hearted bureaucracy, perhaps Brown and Maroudas should give thanks that they don't have to write the pages of scientific detail and justification required for a National Institutes of Health grant, and get on with improving things for the future. And that includes doing everything possible to prevent the wastage of women science graduate students.

BRIGID HOGAN

Department of Cell Biology,

School of Medicine,

Vanderbilt University,

Nashville, Tennessee 37232, USA

\section{Fair shares}

SIR-Britain was once one of the world's wealthiest nations, and many of its worldrenowned scientific establishments and museums can probably trace their origins back to those happy days.

If, as seems to be the case, research groups elsewhere suffer from the slow eclipse of such institutions because of local financial constraints, would it not be logical for those concerned to shoulder a substantial part of their costs?

In other words, why not internationalize the British scientific institutions most at risk, even if this would to some extent dilute their Britishness?

After all, in the last decade of the second millennium, it is the health of world science that matters rather than that of its individual national departments.

CARLO PISCICELLI-TAEGG

Via Ippolito Nievo, 28/A,

20145 Milano, Italy

nuclear membrane subsequently became differentially selective as to which messenger RNAs were permitted to exist, this could supplement other mechanisms that promote cell differentiation and thus true multicellularity. 